SOCIAL RISK, VULNERABILITY AND PRECARITY: EXPLORING MIGRATION AND SETTLEMENT EXPERIENCES IN MID- TO LATER LIFE

K.M. Kobayashi ${ }^{1}$, M. Khan, PhD Candidate ${ }^{2}$, 1. University of Victoria, Victoria, British Columbia, Canada, 2. University of Victoria, Victoria, BC, Canada

As the profile of older adults in the Global North changes with increasing proportions of foreign-born aging populations, there is a need to examine the intersecting markers of immigration, race/ethnicity, cultural beliefs and practices, poverty, and socioeconomic inequality among older immigrants (OI) as they manifest in their experiences of invisibility, marginalization, and exclusion. Despite their demographic significance, there has been little research on the complex and varied experiences of aging, risk, and insecurity among this group, particularly in the context of life course events like migration. A more comprehensive understanding of vulnerability and social risk among OI, as Grenier et al. (2017) point out, necessitates that closer attention be paid to the fragmented lived experiences of those outside the labour force, i.e., recently-arrived OIs. To this end, the framework of precarity is useful as it brings into sharp relief the creation of marginal identities that are often tied to migration.

\section{HOW LIFE COURSE DYNAMICS MATTER FOR PRECARITY IN LATER LIFE}

R.A. Settersten, Jr., Oregon State University, Corvallis, Oregon, United States

This presentation examines precarity and aging through a life course lens. It uses three central dimensions to conceptualize precarity in later life: (1) time (e.g., linked to earlier life periods), (2) domains (e.g., linked to experiences and statuses in education, work, family, health, or wealth), and (3) levels (e.g., linked to individual, relational, institutional, demographic or historical experiences). Empirical illustrations reveal five important lessons: that precarity often (1) has spillover effects across time, domains, and levels; (2) has social origins and consequences, bringing potential malleability and openness to intervention; (3) is felt acutely in and expressed through social relationships, making it an interpersonal phenomenon; (4) is experienced in distinct ways for women and men and members of distinct social groups; and (5) is associated with social inequalities, meaning that some of its causes, as well as protections against it, stem from the welfare state, thus making it a political phenomenon.

\section{SESSION 910 (PAPER)}

\section{COGNITIVE FUNCTIONING II}

\section{THE UNITY AND DIVERSITY OF EXECUTIVE FUNCTIONS ACROSS ADULTHOOD}

\section{B. Katz ${ }^{1}$, A. Ogletree ${ }^{1}$, P. Shah ${ }^{2}$, 1. Virginia Tech, 2.} University of Michigan

Executive function (EF) capacities, such as working memory, cognitive flexibility, and inhibitory control, are both somewhat unitary and separable in nature. Performance on measures of these capacities during aging is often highly correlated with instrumental activities of daily living. The extent to which these capacities change during the course of healthy aging is a salient question. We examine a large cross-sectional sample of $\mathrm{N}=18,894$ individuals aged 18 to 100 from across the United States who completed a set of computerized EF tasks to better understand how the relationship between these measures changes across the lifespan. We included tasks based on n-back working memory, the flanker task, the Stroop task, and other commonly used cognitive assessments related to EF. Using a comparative approach with a variety of methodological techniques, including latent variable analysis, we find that, in general, performance on these tasks is more similar among younger adults and more separable later in life. Specifically, performance on working memory tasks was less likely to be grouped with cognitive flexibility and inhibitory control as a unitary construct in old age. This may be due to variance in growth and declines in different cognitive abilities throughout the lifespan. This work is among the first research to examine the nature of EF using latent variable analysis throughout the lifespan with a large, national sample. We discuss how these findings have important implications for understanding the nature of $\mathrm{EF}$ during aging and how they may highlight which functions should be targeted by cognitive interventions.

\section{THE RELATIONSHIP BETWEEN SMOKING} DURATION AND COGNITIVE FUNCTIONING IN MID- AND LATE-LIFE: A CO-TWIN CONTROL STUDY S. Pahlen, C. Reynolds, University of California Riverside

Smoking and its influence on health is well known but the relation of smoking on cognitive functioning is less understood, with inconsistent findings as to impacts on cognitive functioning in late adulthood. This paper will explore these relations to determine if smoking does impact cognitive performance. To address these questions, the current study will use data collected and harmonized by the international IGEMS (Interplay of Genes and Environment across Multiple Studies; Mage $=66.1$ ) consortium. Measures included smoking duration and cognitive ability assessments in twins from three countries (Sweden, Denmark, and the USA): Digits Backwards, Digits Forward, Symbol Digit, Block Design, and Synonyms. Negative associations were found between smoking duration and most cognitive tasks $(\mathrm{r}<-.16)$, with significant negative effects for Symbol Digit and Block Design, although magnitudes differed by sex. Synonyms demonstrated a significant but positive association $(\mathrm{r}=.11)$ with performance in females. To examine potential causal relationships between smoking duration and cognitive performance, a co-twin control (CTC) design was used, adjusting for age. For males, smoking duration was found to negatively impact Symbol Digit, Synonyms, and Block Design in comparing within-pair twin effects among discordant twins $(\mathrm{d}<$ $-0.15)$; effect sizes were larger for the dizygotic than monozygotic twins, suggesting partial genetic confounding. The within-twin pair effects for females were less consistent in magnitude and direction between monozygotic and dizygotic twins suggesting a complex set of etiologies. Cohort and SES contributions will be examined to evaluate potential mediating and moderating effects. 